Referencia para citar este artículo: Murillo-Oviedo, A. B., Rodrigues de Oliveira Medeiros, C. \& Machado Valadão Júnior, V. M. (2017). La normatividad religiosa en una ONG: Experiencias negociadas de edad y género. Revista Latinoamericana de Ciencias Sociales, Niñez y Juventud, 15(1), pp. 533-545.

\title{
La normatividad religiosa en una ONG: Experiencias negociadas de edad y género*
}

\author{
ANA BEATRIZ MURILLO-OVIEDo ** \\ Estudiante Universidade Federal de Uberlândia, MG, Brasil. \\ Cintia Rodrigues de OLIVEIRA MEDEIRos ${ }^{* * *}$ \\ Profesora Universidade Federal de Uberlândia, MG, Brasil. \\ VALDIR MACHADO VALADÃO JÚNIOR ${ }^{* * * *}$ \\ Profesor Universidade Federal de Uberlândia, MG, Brasil.
}

\section{Artículo recibido en mayo 13 de 2016; artículo aceptado en julio 21 de 2016 (eds.)}

Resumen (analítico): Este artículo se basa en el análisis de la crononormatividad, mediante el análisis de la negociación de género y edad influidos por la religión predominante en la gestión administrativa de una ONG en Uberlândia, MG, Brasil. Nuestro objetivo es explorar el papel de la normatividad religiosa en experiencias negociadas de edad y género, analizando el discurso de las mujeres que forman esta ONG. Realizamos entrevistas con ocho trabajadoras, basadas en la relación entre religión, género y edad en las experiencias laborales. Encontramos que existe sufrimiento y sacrificio por las exigencias aceptadas y ligadas a la condición de mujer, pero también satisfacción, confianza y esperanza por la misión de la organización y la función que las entrevistadas desempeñan.

Palabras clave: Género, edad, religión, crononormatividad (Tesauro: http://skos.um.es/ unescothes/).

\section{Religious normativity in an NGO: Negotiated experiences of age and gender}

- Abstract (analytical): This paper is based on an analysis of chrononormativity, analyzing the negotiation of gender and age as influenced by religion in the management of an NGO in Uberlândia, $M G$, Brazil. The aim of the study is to explore the function of the norms imposed by religious beliefs on the negotiated experiences of age and gender, analyzing the discourses of the NGO's female workers. Eight women were interviewed with the questions based on the relation between religion, gender and age and their experiences working at the NGO. The study identified suffering and sacrifice among the workers due to the demands placed on them due to their condition as women, however they also feel satisfaction, trust and hope thanks to the organisation's mission and the jobs that they hold.

Key words: Gender, age, religion, chronormativity (Tesauro: http://skos.um.es/unescothes/).

\footnotetext{
Reporte de caso. Este artículo surge como iniciativa científica de la disciplina Cultura Organizacional, de la Maestría Académica en Administración, de la Universidad Federal de Uberlândia, Minas Gerais, Brasil. Fue realizada entre abril y julio del 2015. Investigación cualitativa con entrevistas. Área: Ciencias Sociales Aplicadas. Subárea: Estudios de Género.

** Administradora de la Universidad Nacional de Costa Rica. Estudiante de la Maestría en Administración de la Universidad Federal de Uberlândia. Correo electrónico: abmo10@gmail.com

**** Doctora en Administración por la Fundación Getulio Vargas - EAESP. Máster en Administración por la Universidad Federal de Uberlândia. Profesora Ajunta II de los cursos de Grado y Posgrado de la Facultad de Gestión y Negocios de la Universidad Federal de Uberlândia. Correo electrónico: cintia@ufu.br

**** Doctor en Ingeniería de Producción por la Universidad Federal de Santa Catarina, Máster en Administración por la Universidad Federal de Paraná. Profesor asociado de la Universidad Federal de Uberlândia, en cursos de Grado y Posgrado en Administración. Correo electrónico: valdirjr@ufu.br
} 


\section{Ana Beatriz Murillo-Oviedo - Cintia Rodrigues de Oliveira Medeiros - \\ VALDIR MACHADO VALADÃO JÚNIOR}

\section{A normatividade religiosa em uma ONG: Experiências negociadas de idade e gênero}

- Resumo (analítico): Este artigo está fundamentado na análise da crononormatividade, analisando a negociação do gênero e a idade influenciada pela religião dominante na gestão administrativa de uma ONG, em Uberlândia, MG, Brasil. Nosso objetivo é explorar o papel da normatividade religiosa nas experiências negociadas de idade e gênero, analisando o discurso das mulheres que formam a ONG. Realizamos entrevistas com oito funcionárias, com base na relação entre religião, gênero e idade nas experiências laborais. Encontramos que existe sofrimento e sacrifício pelas exigências aceitas da condição de mulher, mas também, satisfação, confiança e esperança pela missão que possui a organização e a função que cada uma desempenha na ONG.

Palavras chave: Gênero, idade, religião, crononormatividade (Tesauro: http://skos.um.es/ unescothes/).

-1. Introducción. -2. Género, edad y religión. -3. Metodología de investigación. -4. Educando en la religión. -5. Discusión de los resultados. -6. Consideraciones finales. -Lista de referencias.

\section{Introducción}

Los estudios de género surgieron basados en el análisis de diferencias en las condiciones que la sociedad establece para la dicotomía hombre-mujer (Acker, 1990, Bruni, Gherardi \& Poggio, 2005), más allá de las características biológicas propias de cada sexo, por tanto, son dinámicas y construidas constantemente (West \& Zimmerman, 1987).

La mujer aparece en una posición de sumisión y minoría frente al hombre (Acker, 1990, Bruni et al., 2005) y su identificación se basa en aquello que no es propio de la masculinidad (Martin, 2001), institucionalizada por el carácter masculino dado a la organización y a su gestión. En esta institucionalización, la religión como sistema cultural (Geertz, 1978) establece normas para la formulación de conceptos y la creación de motivaciones que podrían generar diferencias de género entre los fieles.

Así, siguiendo los trabajos realizados por Acker (1990) y Bruni et al. (2005) sobre la institucionalización del género, y las definiciones sobre "hacer género" (West \& Zimmerman, 1987) y la normatividad (Freeman, 2010) el objetivo de este artículo, explorar el papel de la normatividad religiosa en las experiencias negociadas de edad y género en mujeres trabajadoras de una $\mathrm{ONG}$ de educación infantil. Es en este contexto que las niñas y los niños van construyendo su identidad y reproducen patrones y papeles característicos de la organización familiar latinoamericana, también reflejados en sus juegos (de OliveiraPinto \& de Fátima-Lopes, 2009) y en las formas de aprendizaje (De la Cruz, Huarte \& Sheuer, 2004).

Basados en la metodología utilizada por Riach, Rumens y Tyler (2014), al analizar la crononormatividad en la interacción entre género, edad y sexualidad, analizamos la negociación de género y edad en una $\mathrm{ONG}$, ubicada en Uberlândia, MG, Brasil, orientada por una religión y que, operacionalmente, no incluye hombres. Además, reconocemos la religión como una parte de la vida de las personas, constituida por comportamientos, actitudes, creencias, emociones, pensamientos, experiencias y valores propios a su afiliación religiosa, por tanto, es en los argumentos y percepciones de las entrevistadas donde encontramos la normatividad.

Estructuramos el artículo en cuatro partes después de esta introducción: revisión de literatura sobre investigaciones de edad y género, y la visión de la religión como sistema cultural. Posteriormente, describimos los procedimientos metodológicos, para, en una tercera parte, analizar y discutir los resultados de las entrevistas. Finalmente, presentamos las consideraciones finales y sugerencias para estudios futuros.

\section{Género, edad y religión}

La masculinidad y el patriarcado son elementos predominantes en la organización 
jerárquica de las relaciones familiares y sociales. De esta forma, en las organizaciones se dan relaciones asimétricas de género reproducidas en procesos y patrones preestablecidos, siendo el género una construcción relacional y dinámica. Las características de la feminidad y la masculinidad en las acciones de las personas influyen el "hacer género", como práctica social propia de contextos en los cuales se dan relaciones asimétricas de poder (Murgia \& Poggio, 2009).

Género es el artefacto lingüístico creado por el feminismo, consecuencia del sistema de diferencias sociales legalizadas y objetivadas en diferentes situaciones: práctica social y discursiva de "hacer género", y representación que da significado al individuo; fundamentado en el sistema de dominio de la sociedad, en la repetición de acciones y discursos de prestigio, identidad y estatus (Bruni et al., 2005) en diferentes contextos.

La división del trabajo se manifiesta en cinco procesos que producen estructuras sociales de género: 1) decisiones de gestores promueven divisiones entre género, en puestos de decisión principalmente para hombres; 2) construcción de símbolos e imágenes que explican y refuerzan las divisiones: lenguaje, ideología, cultura y vestimenta; 3) interacciones entre género incluyen patrones de dominio y sumisión; 4) producción de componentes genéricos de la identidad individual, incluyendo la conciencia de la existencia de los otros tres procesos; y 5) género ayuda a la creación y definición de relaciones en estructuras organizacionales y sociales (Acker, 1990).

Los procesos de género son invisibilizados en las organizaciones por la supuesta neutralidad o los discursos concebidos como asexuados, como parte de la cultura organizacional, y sugieren formas en las cuales género, cuerpo y sexualidad son parte del proceso de control y desigualdad (Acker, 1990).

Apesar de que la discusión sobre la asimetría del poder para categorías de género y edad estas predominan en empresas e instituciones, diferenciándose de valores orientadores, objetivos y dinámicas de las organizaciones del tercer sector (Rifkin, 1997, Salamon, 1998, Tenório, 2004), esta expectativa no se cumple en la práctica.
Algunos estudios en Brasil muestran la ausencia de evidencia sobre diferencias asociadas a la percepción de la edad (experiencia y tiempo de trabajo) (Gomes, Machado, Beuren \& Varela, 2011), así como el predominio de las relaciones de poder y la lógica imperialista en relación con lo masculino, lo blanco y lo europeo, de forma que lo femenino y lo viejo son distanciados de espacios de decisión, aún en las ONG (Medeiros \& Valadão Júnior, 2011, Rosa, Medeiros \& Valadão Júnior, 2012, Valadão Júnior, Herck \& Medeiros, 2014), de modo que hay similitudes en la gestión de las divergencias dentro de las organizaciones públicas, privadas y del tercer sector.

Podemos valorar así la creciente preocupación por considerar la edad en las estrategias de recursos humanos en las organizaciones como la evaluación de las ventajas y los beneficios que se obtienen conservando o contratando personal experimentado, disminuyendo la discriminación por edad, género y raza, entre otros (Jorgensen, 2005).

Desde el punto de vista de los gestores, Riach (2009) analiza discursos sobre estrategias para identificar la diversidad y promover la consideración de la edad en la búsqueda de organizaciones igualitarias, considerando la normatividad social. Por su parte, Gellner, Schnieder y Veen (2011) analizan el desempeño basado en dos dimensiones: calidad y cantidad, de forma que la edad afecta la segunda, pero mejora la calidad del desempeño.

Divisiones de género asociadas a la edad se presentan también en las organizaciones. En el caso de los hombres, pueden revalorar sus funciones en la sociedad mientras adquieren más experiencia y edad. Sin embargo, para las mujeres hay dificultades internas y externas para lidiar con la edad, establecidas en la normativa social, pues a partir de los 40 años, se considera que están "viejas" para trabajar, y su conocimiento no es valorado (Jyrkinen \& McKie, 2012).

De esta forma, edad y género forman parte de las definiciones culturales de las organizaciones, y se crean significados comunes para cada miembro, con influencia de 
la ideología o religión, cuando forman parte del sistema cultural y administrativo dominante de una organización.

La religión tiene funciones reguladoras de la vida social, como sistema cultural o como razón práctica de los mandatos divinos. Establece un "ritmo cronológico" para los fieles, más allá de otras reglas que delimitan acciones, actividades y decisiones en la vida; entre las reglas se encuentran también costumbres y principios morales establecidos sin que la persona pueda percibirlo, identificarlo o cuestionarlo (Usarski, 2010).

Por tanto, la religión se establece como normativa al ser un sistema cultural simbólico, que crea conceptos y definiciones percibidas como reales por medio de disposiciones $\mathrm{y}$ motivaciones que penetran en las actividades de las personas y perduran por estar basadas en hechos considerados como poderosos y reales (Geertz, 1978). No obstante, la influencia de la religión puede ser la solución para problemas diarios y proveer mejores condiciones de vida para sus miembros, principalmente las mujeres (Hernández \& Burguete, 2005, Lindhardt, 2012).

Algunos estudios que analizan la relación entre género y religión tienen una vertiente relacionada con la práctica de la religión, y considera que las mujeres son más religiosas en sus actitudes, valores y conductas (Walter \& Davie, 1998). Una vertiente utiliza resultados del World Values Survey, que establece diferencias de religiosidad como producto de las diferencias en la socialización (Miller \& Stark, 2002), o compara la religiosidad entre hombres y mujeres en el judaísmo y el islamismo. Se excluye a las mujeres de las posiciones de liderazgo, y las separa en prácticas y rituales religiosos. Así, se establecen normas que reafirman la identidad y los ideales de la masculinidad religiosa (Sullins, 2006).

De acuerdo con Collett y Lizardo (2009), el género de una persona no influye en su religiosidad, como sí lo hace la orientación femenina. Esta orientación puede ser un trazo social permanente, presente en varios niveles de la persona, y ligado a patrones de religiosidad establecidos (Thompson Jr., 1991,
Francis, 1997). En esta forma, la cultura y la tradición de cada religión diferencia las tareas religiosas que corresponden a hombres y mujeres (Lowenthal, Macleod \& Cinnirella, 2001, Modiri \& Azadarmaki, 2013), lo que influye normativamente las experiencias de vida de cada género.

Las iglesias Pentecostales en Chile establecen la capacidad de liberar, controlar $\mathrm{u}$ oprimir a sus miembros, y son capaces de influir en espacios privados como las relaciones familiares y de género, a partir de las organizaciones y los roles establecidos en sus principios (Lindhardt, 2012).

En textos de la tradición cristiana, la construcción social de género en instituciones sociales, como la familia, el estado, las organizaciones de educación y el trabajo, la religión, etc. establece diferencias institucionalizadas de género que definen que la mujer debe servir y vivir para el otro, como principio natural que está presente en espacios públicos y privados de su vida (Souza, 2007). Esto se traduce en representaciones domesticadoras que implican "ser buena madre, esposa, ama de casa y religiosa fiel [...] fragilidad, sumisión y cuido (con hijos, marido y en las actividades domésticas)" (p.19).

Para analizar la función de la religión, Dockendorff (2015) parte de la visión de Habermas sobre el pensamiento social que promueve la religión como un proceso autoreflexivo para conseguir un ideal humano social. En esta forma, las tradiciones religiosas facilitan aquello que no está en la sociedad, como intuición moral, creando conciencia social sobre lo correcto y lo incorrecto, impulsando cambios y mejoras constantes, y proporcionando la base para la vida pública.

Religión, edad y género convergen en las organizaciones reafirmando o creando identidades basadas en sistemas, creencias y prácticas reproducidas. La investigación que realizamos, considera los condicionantes religiosos que influyen en las percepciones de las mujeres de una ONG de base religiosa, por medio de la siguiente metodología. 


\section{Metodología}

La metodología utilizada por Riach et al. (2014), para des/construir la crononormatividad en la negociación de edad, género y sexualidad en la vida organizacional es la guía utilizada para el análisis de la negociación de género y edad por medio de la normatividad religiosa en una $\mathrm{ONG}$.

Teóricamente, las ONG están guiadas por un objetivo social que recibe la influencia de las ideologías y la cultura de sus fundadores. En su comienzo, en Brasil, "se produjo una convergencia entre la noción de 'comunidad' como lugar de trabajo educativo, inherente a la educación popular y la 'comunidad' como nueva forma de ser Iglesia en la base" (Oliveira, 1999, p. 49), formando así una red de conocimientos con líderes comunales, en relaciones de poder que suponen isonomía, es decir, la idea de simetría en el poder (Rifkin, 1997; Salamon, 1998; Tenório, 2004).

Sin embargo, incluso en las actividades de emprender y liderar procesos sociales, se dan diferencias entre géneros, pues se asume que el emprenderismo es una habilidad de la masculinidad (Bruni et al., 2005) y que es parte de la cultura organizacional. No obstante, el empoderamiento es una construcción histórica para hombres y mujeres (Muñoz \& Pazos, 2015). En las ONG, la cultura de los fundadores y los gestores influye en la organización y en las identidades que se forman y se negocian en ella, incluyendo características propias al concepto de ONG: innovación, espontaneidad y diversidad, estructura ágil y flexible, y capacidad de improvisación (Oliveira, 1999). Una ONG basada en principios religiosos actuará de acuerdo con los valores que fundamentan su existencia, incluso las formas de trabajo de sus miembros.

En este contexto, las mujeres que forman una ONG son la base de este trabajo, con el fin de conocer cómo su concepción de género y edad es negociada con la normativa religiosa establecida, según su percepción; motivo por el cual no realizamos análisis de documentos organizacionales. Para conocer la dinámica entre género, edad y normatividad religiosa, entrevistamos ocho mujeres, de edades entre 24 y 53 años, que trabajan en una ONG de educación infantil. La organización atiende 122 niñas y niños hasta los cuatro años de edad, ubicada en la ciudad de Uberlândia, Minas Gerais, Brasil. Esta ONG está afiliada a una iglesia que proporciona el $50 \%$ de los recursos necesarios para funcionar.

Este análisis cobra importancia por el papel de la ONG en la formación de niñas y niños, en sus procesos de socialización y construcción de identidad a partir las experiencias vividas en la escuela y aprendidas con sus maestras (Mieles \& García, 2010).

Realizamos las entrevistas en julio de 2015. Cada una decidió el nombre utilizado para describir los resultados, con el objetivo de mantener la confidencialidad de su testimonio.

Para iniciar la entrevista, solicitamos que analizaran, sobre un diagrama de Venn, las relaciones y conexiones existentes entre los tres conceptos, para después profundizar las experiencias vividas en relación con la percepción de edad y género a partir de la religión.

En el guión, y para identificar las relaciones entre los tres conceptos (género, edad, religión) tomamos como base las preguntas utilizadas en la investigación de Riach et al. (2014): 1) sobreposiciones, conexiones, contradicciones y oposiciones; 2) experiencias de trabajo presentes, pasadas y futuras; 3) cambios en las experiencias; 4) cambios en la percepción propia a lo largo del tiempo.

Cada entrevista duró 30 minutos, aproximadamente, y fueron realizadas en el lugar de trabajo, en un espacio que facilitara la conversación. Transcribimos cada entrevista, lo que produjo 40 hojas de transcripción para el análisis de contenido.

El Cuadro 1 caracteriza las entrevistadas, seleccionadas por conveniencia, en las funciones de profesoras, educadoras, cocinera y servicios generales. Debido a que la organización tiene únicamente una secretaria y una directora, éstas fueron seleccionadas automáticamente. 
Cuadro 1. Descripción de las Entrevistadas.

\begin{tabular}{|c|c|c|c|c|c|c|}
\hline Nombre & Función & $\begin{array}{c}\text { Años } \\
\text { aproximados de } \\
\text { trabajar en la } \\
\text { ONG }\end{array}$ & $\begin{array}{l}\text { Edad (en } \\
\text { años) }\end{array}$ & Estado civil & $\begin{array}{l}\text { Tiene } \\
\text { hijos }\end{array}$ & $\begin{array}{c}\text { Cursó o está } \\
\text { cursando } \\
\text { alguna carrera } \\
\text { universitaria }\end{array}$ \\
\hline Gabi & Profesora & 6 & 45 & Casada & $\mathrm{Si}$ & No \\
\hline Erica & Profesora & 1 & 30 & Casada & $\mathrm{Si}$ & $\mathrm{Si}$ \\
\hline Leilda & Educadora & $2 y$ tres meses & 30 & Casada & $\mathrm{Si}$ & $\mathrm{Si}$ \\
\hline Raquel & Educadora & 4 & 33 & Soltera & No & No \\
\hline Jasmine & Cocinera & 4 & 48 & Casada & $\mathrm{Si}$ & No \\
\hline Carmen & Secretaria & 12 & 53 & Casada & $\mathrm{Si}$ & $\mathrm{Si}$ \\
\hline Ana Clara & $\begin{array}{l}\text { Servicios } \\
\text { Generales }\end{array}$ & Tres meses & 24 & Casada & $\mathrm{Si}$ & No \\
\hline Oidinha & Directora & 18 & 53 & Casada & $\mathrm{Si}$ & $\mathrm{Si}$ \\
\hline
\end{tabular}

Fuente: Elaborado por los autores.

Analizamos las entrevistas siguiendo las recomendaciones de Bardin (1977), revisando el material para hacer un pre-análisis e identificar los elementos comunes, a partir de las categorías principales que fueron definidas con los puntos guía del trabajo de Riach et al. (2014) de la siguiente forma: 1) Relaciones entre género, edad y religión; 2) Experiencias de trabajo y cambios en relación con la edad. Además, conforme la literatura analizada, identificamos características, discursos o prácticas relacionadas con un ritmo cronológico y normativo establecido por la religión o por el género, para analizar los puntos de contacto entre género, edad y religión que limitan o favorecen el desarrollo de las mujeres que trabajan en el centro educativo estudiado.

\section{Educando en la religión: Negociando género y edad en una ONG por medio del amor}

Religión, género y edad están relacionados en la dinámica organizacional de la ONG que estudiamos, y en esta relación se expresa dedicación, convicción y resignación con las condiciones vividas (Hernández \& Burguete, 2005, Lindhardt, 2012). Los resultados están fundamentados en el testimonio de las entrevistadas.

\subsection{El ritmo de la religión}

La religión impacta la visión de mujer y define su forma de actuar, vivir y enfrentar las pruebas de la vida. Al ser normativa en la ONG y en la vida de las mujeres proporciona herramientas y valores espirituales como el amor: "...la base de todo es el amor. Y eso se liga completamente con religión, ¿verdad? Ya que Jesús es amor, entonces, por mi religión, eso es todo, el amor" (Ana Clara, 2015).

Las reglas establecidas en una religión (Lowenthal et al., 2001, Modiri \& Azadarmaki, 2013) son transmitidas con referencia a una persona o un grupo que representa la experiencia viva de los mandatos de la creencia. Existe una reinterpretación en la vida de cada miembro, para seguir los comportamientos y las actitudes propios de la normativa definida y transmitida desde la infancia (Usarski, 2010). Aunque en esta ONG no se reconozca la existencia de una normativa como tal, pues no fue identificado por las entrevistadas, la religión es transmitida desde la infancia como parte de la socialización primaria de las funcionarias de la ONG, y de las niñas y los niños que asisten a esta $\mathrm{ONG}$.

“... desde que yo era niña, mi mamá me enseñó (...) y me ayudó mucho, mi religión, porque así, creo que me formó así como una persona mejor, en mi manera de ser. Vamos a suponer ahora 
que una persona no conozca la Palabra, que no conozca la religión ni nada, tal vez no tiene aquella, aquella manera de ser, que una persona que sí tiene a Jesús (...) Muchas veces una persona que no tiene religión no lo aprende desde pequeño y no sabe y va hace mal..." (Jasmine, 2015).

La función normativa de la religión (Habermas, citado por Dockendorff, 2015) está reforzada por la función educadora de esta $\mathrm{ONG}$, defendida por sus trabajadoras, desde aquellas que están a cargo de la enseñanza, hasta las que están en funciones de servicios generales o cocina: “... todo es religión, los niños desde que son bebés aprenden a orar, aprenden a agradecer al Padre, aprenden, verdad, a respetar todo con base en la religión..." (Ana Clara, 2015).

Así mismo, sin que fueran definidas normas específicas dentro de la ONG, hay un reconocimiento sobre las reglas de cómo debe actuar una persona creyente, y se consideran una guía para la vida de las personas, tanto dentro como fuera de la $\mathrm{ONG}$, así como para el papel de la mujer en la sociedad,

"... las personas se basan en la Biblia, y la propia Biblia es la que da los fundamentos para, ¿verdad? ella orienta todo, por ejemplo como usted se relaciona con las personas... orienta para tener presupuestos, y principalmente así, de la mujer, habla mucho de la sabiduría de la mujer..." (Raquel, 2015).

“... mi base es la religión, ¿sabe?, como me tengo que portar, como madre, como mujer, como ser ciudadana, respetar las leyes, porque en mi religión, incluso en la Palabra dice que Jesús cumplía Su ley y la ley de los hombres" (Ana Clara, 2015).

Como parte de las funciones dadas por la religión, pero principalmente para la mujer, como lo analiza Souza (2007), la vocación de servicio al otro, mansedumbre y dominio propio están presentes en el discurso de estas mujeres, parte fundamental de su manera de ser. De esta forma, encontramos que existe una percepción de responsabilidad de la mujer por el bienestar de su entorno, así como por la función de ser soporte para el matrimonio, bajo el discurso de que:
"Quien hace y sustenta, yo digo que primero es Dios dentro del matrimonio, pero quien hace que un matrimonio perdure es la esposa, si usted es una mujer explosiva, se termina, nosotras tenemos que ser mansas" (Oidinha, 2015).

Así, la religión como parte del proceso de formación y educación de la persona reproduce reglas, valores y acciones que determinan las formas como estas mujeres llevan su vida familiar y profesional.

\subsection{La relación género-religión}

La esperanza y el amor, como valores que definen la vida de las mujeres en esta ONG, parten de la creencia en Dios y de la práctica de la religión (Walter \& Davie, 1998). Así, edad y género están en constante análisis, como crecimiento espiritual.

La ONG está formada únicamente por mujeres, reforzando esta dicotomía con la institucionalización del género (West \& Zimmerman, 1987), pues definen que el papel de la educadora infantil debe ser de la mujer, al transmitir valores y generar sentimientos en los otros que, de acuerdo con las entrevistadas, solamente ocurre cuando las mujeres son las encargadas de la educación, reforzando la "referencia a las obligaciones" de enseñar que 'corresponde' a la mujer (De la Cruz et al., 2004, p. 45):

"Mujer, ella es más cariñosa con los niños, los padres se sentirían inseguros, creo que los padres tienen más confianza en la mujer que en el hombre, con el cuidado en las acciones, creo que el hombre es más torpe..." (Carmen, 2015).

"Yo creo que un hombre aquí sería muy extraño, ¿sabe? un hombre cuidando un niño, no, no tiene nada que ver, (...) tiene menos paciencia, incluso con los niños. Parece que la mujer ya viene con ese don..." (Ana Clara, 2015).

Consecuentemente, el hombre tiene el papel de protector, función paternalista de Collinson y Hearn (1994), aquel que da seguridad y que puede hacer todo, pues, en este caso, es considerado para la función de portero o servicios generales: 
"Yo pedí a la dirección que pusieran un hombre en servicios generales, porque aquí es así, tal vez las muchachas tienen que sacar la basura para la calle, cambiar un bombillo, hay días que tenemos que llamar a alguien para que cambie los bombillos, o porque hay un problema en los baños, en la cocina, no sé dónde más, ¿sabe? Por eso yo digo que se necesita un hombre..." (Oidinha, 2015).

Esta diferenciación es reforzada por la dirección de la ONG, formada por cuatro hombres y dos mujeres, quienes, como órgano superior de decisiones, impiden la contratación fija de hombres en la organización, justificando que un hombre en el medio de mujeres "no va a funcionar" (Oidinha, 2015), ni para labores de servicios generales, ni para profesor.

Por tanto, la institucionalización de las diferencias de género lleva a las mujeres de esta ONG a la aceptación de una función que les implica cargar más de lo que consideran justo, creyendo que son las únicas responsables por el orden y el cuidado de la casa, los hijos y el marido. La aceptación no se cuestiona, sea que se sientan cansadas o no, siempre es visto como "un papel que viene de allá arriba", e incluso aunque sea difícil lidiar con estas tareas y sientan "ganas de independencia" (Erica, 2015):

“...jPor Dios! La mujer tiene tantas actividades que hacer en casa, que debería haber una ley para trabajar solo medio tiempo, porque las tareas en la casa son también una difíciles, hay que darle atención al marido, al hijo, a la casa, ¿verdad? Y, además, hay que ser administradora del dinero, las compras, las cosas que faltan y las cosas que se necesitan, me parece que es muy difícil ser mujer, es complicado, me parece una responsabilidad muy grande..." (Gabi, 2015).

"Uno crece sabiendo que eso es así, el papel de la mujer es el de cuidar a los hijos, quedarse en casa, todo. Con el tiempo eso ha venido modernizándose (...) pero a mí me parece que es lo adecuado que la mujer sea sumisa a su marido..." (Erica, 2015).
Esta situación se ve compensada por la misión de formación de buenos ciudadanos, analizada por Habermas (citado por Dockendorff, 2015) que es parte de las normas religiosas que son trasladadas para el espacio social.

Al analizar el impacto que el trabajo en la $\mathrm{ONG}$, fundamentada en creencias de una iglesia, tiene en la percepción de género y edad, encontramos que proporciona a las trabajadoras la visión de que están allí para cumplir su misión, y Dios es el mediador de su experiencia, por tanto deben cumplir con la responsabilidad dada.

El reconocimiento de las diferencias de género es evidente en la $\mathrm{ONG}$, pero es justificado como parte de la misión y la religión. Aunque el cansancio y las diferencias estén presentes en las actividades de la casa y del trabajo, confirmando la relación de subordinación mostrada en los trabajos de Medeiros y Valadão Júnior (2011), Rosa et al. (2012) y Valadão Júnior et al. (2014), la religión y el género determinan muchas experiencias vividas a lo largo de la vida de las mujeres de esta organización.

\subsection{Experiencias laborales y cambios en relación con la edad}

La edad como factor generador de experiencia (calidad) pero agotamiento físico (cantidad) (Gellner et al., 2011) está presente en las mujeres de mayor edad en esta ONG, resaltando que cambiarían algunas decisiones en su vida:

“... yo me arrepiento de haberme quedado mucho tiempo sin hacer nada, en la casa solo cuidando de los hijos, ¿sabe?, entonces ese tiempo, no me arrepiento de haber dejado de trabajar para cuidar a mis hijos, pero en cuestión de estudios sí, porque pude haberlos cuidado y estudiar a la vez" (Carmen, 2015).

La visión de la vida y el orden para alcanzar las metas de las entrevistadas puede cambiar de acuerdo con la edad y los logros obtenidos por cada una, sin dejar de lado el cumplimiento de la misión 'encomendada por Dios', proveniente de la visión normativa de la religión. En esta forma, en los casos analizados, hay mujeres 
que trabajaron desde niñas porque su condición socioeconómica lo requería, antes de estudiar o formar una familia, lo que produce inseguridad en sus posibilidades de profesionalización $\mathrm{y}$ en su valoración como mujer, o que no se profesionalizaron conscientemente:

"Yo no le encontraba sentido a hacer una carrera, para tener una profesión. Terminé mi secundaria e hice un curso del magisterio. Y en esa área quedé por miedo de trabajar, entonces fui a trabajar a una ONG que una amiga estaba abriendo, fui ahí y me quedé seis años. De ahí fui a trabajar de empleada doméstica, niñera, niñera, porque tuve mucho miedo de enfrentar la realidad, la vida, incluso hasta intenté hacer una prueba para entrar al servicio público, pero no la hice, me inscribí, pero tuve miedo, había demasiada competencia, tenía que estudiar, no tuve coraje de ir" (Gabi, 2015).

Con el conocimiento de lo vivido, las entrevistadas identificaron que podrían haber tomado decisiones diferentes en su vida, y esas experiencias quedan como alternativas y recomendaciones para otras personas, sin identificar acciones que puedan adelantarse en la vida personal, pues la realización profesional propia está en segundo plano, después del cumplimiento de las funciones de madre $\mathrm{y}$ esposa,

"Una cosa que no yo hice y tengo la certeza que no voy a hacer, dentro de mí ya sé que no podré, es terminar mis estudios... yo comencé el primer año de contabilidad y lo dejé, y ya no tengo ganas de regresar, simplemente, ¿sabe? Yo sé que no..." (Jasmine, 2015)

La edad es percibida como una ventaja por la sabiduría y la madurez profesional y espiritual que adquieren, "cuanto más vieja, tengo más paciencia para el otro" (Carmen, 2015). La experiencia adquirida a través de los años, es valorada por esta organización, reteniendo a trabajadoras con más de 50 años que realizan adecuadamente su trabajo.

No obstante, la edad puede obstaculizar el logro de deseos particulares como estudio y esparcimiento, pues la función de madre y ama de casa está antes que la realización personal y, con mayor edad, consideran que las responsabilidades aumentan, limitándoles la disponibilidad de tiempo. Asimismo, la edad es vista como ejemplo de desgaste en el desempeño profesional, pues como Gabi (2015) mencionó “con esta edad, si salgo de aquí, no será fácil encontrar trabajo, solo si fuera de empleada doméstica". Esta posición es abiertamente discriminatoria.

Por su parte, la administración de la $\mathrm{ONG}$ facilita que esta misión de mujer con trabajo digno sea cumplida, pues, sin importar la edad o la formación, han dado oportunidad de crecimiento y desarrollo a las mujeres que trabajan en ella, facultando y dando confianza para las acciones de cada una dentro de la organización. Esa confianza es parte de la vocación y de las oportunidades para crecer en la ONG; la edad es vista como parte del sistema cultural religioso, y representa la posibilidad de obtener más sabiduría, más experiencia y madurez, tanto profesional como espiritualmente, lo que disminuye el temor por la discriminación por edad o género, y se aceptan los sacrificios y el sufrimiento que puede traer el cumplimiento de su función (Hernández \& Burguete, 2005; Lindhardt, 2012).

Las experiencias de vida de las entrevistadas están marcadas por entrega y dedicación al prójimo, dejando "lo mío para después" (Gabi, 2015), como parte de la misión cristiana, y por la institucionalización social de una perspectiva y de la función desigual de la construcción de género. Por tanto, el papel de la mujer en la sociedad, llevado a todos los ámbitos de la vida, impacta las experiencias laborales, personales y profesionales a lo largo de sus vidas.

\section{Discusión de los resultados}

"Hacer género" (Murgia \& Poggio, 2009), como construcción relacional y dinámica de la sociedad, define las organizaciones reproduciendo patrones como prácticas sociales generalizadas, a partir de discursos legitimados por sistemas culturales como la religión. En los casos analizados en esta ONG, entendimos que la identidad de género es parte de las normas religiosas, pues influye en las funciones 
que la mujer tiene en la sociedad (Miller \& Stark, 2002, Modiri \& Azadarmaki, 2013). En esta forma, la crononormatividad (Riach et al., 2014) o el ritmo cronológico (Usarski, 2010), relacionado con la religión, define las oportunidades de crecimiento y desarrollo profesional y personal de las mujeres, en ocasiones incluso restringiéndolo (Lindhart, 2012). Asimismo, en el papel educacional de la ONG, la construcción del género está formando en las niñas y los niños patrones de identidad, "valores, costumbres, cosmovisiones y formas de relación" (Mieles \& García, 2010, p. 818) que influirán en su comportamiento.

La distinción de género es parte de las acciones, los discursos y las representaciones de la mujer, y suscita múltiples significados basados en el sistema de dominio de la sociedad o de la religión (Bruni et al., 2005). Esta distinción funciona como determinante de las posiciones y las identidades del hombre y de la mujer en la sociedad, y reproduce las diferencias (Acker, 1990), como ocurre en la falta de cuestionamiento de la presencia de hombres en la ONG.

Además, la distinción es replicada por medio de estructuras sociales de género presentes en las decisiones de los gestores (Dirección de la ONG), en la construcción de símbolos como el lenguaje y la cultura (las mujeres son más adecuadas para cuidar niños), en las interacciones entre género (hombres entre mujeres no funcionarían), en los patrones de dominio (es un mandato de Dios), y en los componentes de la identidad individual (es el papel de la mujer) (Acker, 1990).

Los trazos de representaciones domesticadoras de la religión citadas por Souza (2007) están presentes en la descripción de la mujer en las entrevistadas: buena madre, esposa dedicada, ama de casa responsable y religiosa. Estas funciones se caracterizan por la fragilidad, sumisión y cuido del hombre, que 'deben' estar presentes en cualquier actividad.

Según los hallazgos de Gellner et al. (2011), la edad es percibida como una ventaja en relación con la experiencia adquirida, pero disminuye el desempeño, pues además del envejecimiento físico y mental, el cansancio del cuerpo es mayor por el agregado 'papel de ama de casa, esposa y madre'.
Género, edad y religión coinciden en un ambiente de mujeres que perciben ganancias y pérdidas en su vida solo por ser mujeres. La perspectiva de religión proporciona fundamentos para el sacrificio realizado por estas mujeres, al ponerse en segundo plano, dejando pasar deseos $\mathrm{y}$ anhelos personales $\mathrm{y}$ profesionales. Valores que son reproducidos a diario frente a las y los estudiantes.

\section{Consideraciones finales}

La ONG analizada, con base religiosa, presenta flexibilidad $y$ formas de control menos rígidas en la práctica, promoviendo mayor disposición por procesos de aprendizaje colectivos, aceptando ideales de otros y construyendo formas mejores para realizar sus actividades. Sin embargo, esta constatación no se lleva a cabo en la gestión administrativa de la dirección, en la cual es notoria la rigidez en la convivencia del hombre y la mujer en la organización, como reflejo de las formas de diferenciar géneros e institucionalizar esas formas (Acker, 1990, West \& Zimmerman, 1987).

De acuerdo con nuestro objetivo, explorar el papel de la normatividad religiosa en las experiencias negociadas de edad y género en una ONG, y guiados por la metodología de Riach et al. (2014), enfocado en el análisis de las entrevistas con mujeres que trabajan en la organización, encontramos que día a día definen su identidad con base en la función que la religión como sistema definió para la mujer: madre, esposa y ama de casa, sumisa, frágil y cuidadosa, que pone en segundo plano sus objetivos de vida, para cuidar primero la vida de otros (Souza, 2007), creando y defendiendo la normativa percibida por la religión (Lowenthal et al., 2001, Modiri \& Azadarmaki, 2013). En esta forma, el compromiso de estas mujeres con su función de madre hace que, aun siendo jóvenes o con edad media, sus familias y los deseos de sus esposos sean una prioridad, frente a sus deseos de crecimiento personal y profesional, para después darse cuenta, con el paso del tiempo, de que no tienen más oportunidad de realizar sus anhelos. 
En este sentido, el artículo apoya los hallazgos del estudio latinoamericano de Jyrkinen y McKie (2012), en el cual las responsabilidades de cuidar a las personas cercanas a la mujer limitan y atrasan su desarrollo profesional para garantizar primero que el esposo esté bien atendido, que su casa esté en orden y que sus hijos estén bien educados. Tanto así que Gabi sugiere una ley para que el trabajo en casa sea reconocido, pues, como ya Souza (2007) lo analizó, es parte de la normatividad religiosa, pues describe esa función doméstica de la mujer de cuidar a los hijos, al esposo y su casa.

El ambiente y la gestión de la ONG favorecen la percepción de la edad y del género, pues no se presenta discriminación con la mujer ni con su edady, por el contrario, la contratación de las personas se da siendo jóvenes o con más de 40 años. No obstante, se presenta una separación con el hombre, dejándolo solo con la función de ser protector, y el poder que se manifiesta en las relaciones familiares de las mujeres entrevistadas. De esta forma, a pesar de los avances sociales, la gestión en esta ONG genera limitaciones a sus trabajadoras, ofreciéndoles espacios para crecer, pero marcando límites en relación con el género (Lindhardt, 2012), aspectos que sutilmente son pasados a las niñas y los niños que asisten a la institución, al convivir educacionalmente solo con mujeres (Mieles \& García, 2010).

El estudio presenta limitaciones. Aunque el espacio en el que realizamos las entrevistas fuera privado, las entrevistadas parecían cohibidas para dar detalles sobre sus relaciones de trabajo, como uno de los procesos que producen estructuras sociales de género citados por Acker (1990).

Como futuros estudios, recomendamos profundizar, con análisis más exhaustivo de edad y género realizado con organizaciones no gubernamentales, gubernamentales y empresas privadas para conocer si el comportamiento presentado en este caso es parte de la visión ONG como estructura social, de las organizaciones de la sociedades en general o es parte de los valores propios de las ONG con fundamento religioso. El análisis de la percepción por grupos de edad que identifican las percepciones generacionales puede ser investigado. Resaltamos la importancia de analizar el papel de la educación como función social en diferentes niveles: familia, escuela, sociedad, y cómo esa función puede o no estar influida por la presencia femenina en las etapas de formación. Sugerimos estudios internacionales sobre las representaciones sociales de la mujer en el trabajo, así como esa misma construcción es realizada de acuerdo con la religión profesada.

\section{Lista de referencias}

Acker, J. (1990). Hierarchies, Jobs, Bodies: A Theory of Gendered Organizations. Gender and Society, 4 (2), pp.139-158. Doi: 10.1177/089124390004002002.

Bardin, L. (1977). Análise de Conteúdo. Lisboa: Edições 70.

Bruni, A., Gherardi, S. \& Poggio, B. (2005). Gender and Entrepreneurship. An ethnographical approach, London: Routledge.

Collett, J. L. \& Lizardo, O. (2009). A PowerControl Theory of Gender and Religiosity. Journal for the Scientific Study of Religion, 48 (2), pp.213-231.

Collinson, D. L. \& Hearn, J. (1994). Naming Men as Men: Implications for Work, Organization and Management. Gender, Work and Organization, 1, pp. 2-22. Doi: 10.1111/j.1468-0432.1994.tb00002.x.

De la Cruz, M., Huarte, M. F. \& Scheuer, N. (2004). Huellas tempranas del género en la adquisición y transmisión cultural: concepciones de niños y niñas sobre el aprendizaje de la escritura. Revista Latinoamericana de Ciencias Sociales, Niñez y Juventud, 2 (1), pp. 67-86.

de Oliveira-Pinto, T. \& de Fátima-Lopes, M. (2009). Brincadeira no espaço da rua e a demarcação dos gêneros na infância. Revista Latinoamericana de Ciencias Sociales, Niñez y Juventud, 7 (2), pp. 861885.

Dockendorff, C. (2015). Pretensiones normativas de la religión como problema. Una aproximación desde las teorías sobre secularización y diferenciación social de 
Habermas y Luhmann. Revista de Estudios Sociales, 51, pp. 36-49. Doi: 10.7440/ res51.2015.03.

Francis, L. J. (1997). The psychology of gender differences in religion: A review of empirical research. Religion, 27 (1), pp.8196.

Freeman, E. (2010). Time binds: Queer temporalities, queer histories. Durham: Duke University Press.

Geertz, C. (1978). A religião como sistema cultural. En C. Geertz. A interpretação das Culturas, (pp. 107-135). Rio de Janeiro: Zahar.

Gellner, U., Schneider, M. \& Veen, S. (2011). Effect of Workforce Age on Quantitative and Qualitative Organizational Performance: Conceptual Framework and Case Study Evidence. Organization Studies, 32 (8), pp. 1103-1121. Doi: 10.1177/0170840611416746.

Gomes, G., Machado, D. G., Beuren, I. M. \& Varela, P. S. (2011). Gênero e estilo de gestão: Um estudo em Organizações Não Governamentais (ONGs) brasileiras. Sociedade, Contabilidade e Gestão, 6 (2), pp.37-53.

Hernández, G. P. R. \& Burguete, J. L. C. (2005). Religión y dinámica familiar en Los Altos de Chiapas. La construcción de nuevas identidades de género. Estudios sociológicos, 23 (68), pp. 515-534.

Jorgensen, B. (2005). The ageing population and knowledge work: A context for action. Foresight, 7 (1), pp.61-76. Doi: 10.1108/14636680510581321.

Jyrkinen, M. \& McKie, L. (2012). Gender, age and ageism: Experiences of women managers in Finland and Scotland. Work Employment \& Society, 26 (1), pp. 61-77. Doi: 10.1177/0950017011426313.

Lindhardt, M. (2012). Poder, género y cambio cultural en el pentecostalismo chileno. Cultura y Religión, 3 (2), pp. 94-111.

Lowenthal, K. M., Macleod, A. K. \& Cinnirella, M. (2001). Are Women more Religious than Men? Gender Differences in Religious Activity Among Different Religious Groups in the UK. The Journal of Personality and Individual Differences, 32, pp. 139-133.

Martin, P. Y. (2001). Mobilizing Masculinities': Women's Experiences of Men at Work. Organization, 8 (4), pp. 587-618. Doi: 10.1177/135050840184003.

Medeiros, C. R. \& Valadão Júnior, V. M. (2011). Masculinidade e feminilidade na Ameas: Holograma, ilhas de clareza ou uma selva desconhecida? Cadernos Ebape. BR, 9 (1), pp. 79-96.

Mieles, M. D. \& García, M. C. (2010). Apuntes sobre socialización infantil y construcción de identidad en ambientes multiculturales. Revista Latinoamericana de Ciencias Sociales, Niñez y Juventud, 8 (2), pp.809819.

Miller, A. \& Stark, R. (2002). Gender and Religiousness: Can Socialization Explanations Be Saved? American Journal of Sociology, 107 (6), pp. 1399-1423. Doi: 10.1086/342557.

Modiri, F. \& Azadarmaki, T. (2013). Gender and Religiosity. Journal of Applied Sociology, 24 (3), pp. 1-14.

Muñoz, S. C. C. \& Pazos, L. S. (2015). Empoderamiento de mujeres de una $\mathrm{ONG}$ colombiana. Un estudio de caso simple. Revista de Administração Pública, 49 (6), pp. 1597-1618.

Murgia, A. \& Poggio, B. (2009). Challenging Hegemonic Masculinities: Men's Stories on Gender Culture in Organizations. Organization, 16 (3), pp. 407-423. Doi: 10.1177/1350508409102303.

Oliveira, M. (1999). Globalização e Cidadania: a política externa brasileira e as ONGs. Brasília: Funag.

Riach, K. (2009). Managing 'difference': Understanding age diversity in practice. Human Resource Management Journal, 19 (3), pp. 319-335, Doi: 10.1111/j.17488583.2009.00096.x.

Riach, K., Rumens, N. \& Tyler, M. (2014). Un/ doing Chrononormativity: Negotiating Ageing, Gender and Sexuality in Organizational Life. Organization Studies, 35 (11), pp. 1677-1698. Doi: 10.1177/0170840614550731. 
Rifikin, J. (1997). Identidade e natureza do Terceiro Setor. Em E. B. Ioschpe (ed.) $3^{o}$ setor. Desenvolvimento Social Sustentável, (13-23). São Paulo: Paz e terra.

Rosa, A. R., Medeiros, C. R. \& Valadão Júnior, V. M. (2012). Sob as sombras do discurso colonial: Subalternidade e configurações de gênero em uma lavanderia do interior de Minas Gerais. Cadernos Ebape. BR, 10 (2), pp. 393-410.

Salamon, L. (1998). A emergência do Terceiro Setor-uma revolução associativa global. Revista de Administração, 33 (1), pp. 5-11.

Souza, S. (2007). Violência de gênero e religião: Alguns questionamentos que podem orientar a discussão sobre a elaboração de políticas públicas. Revista Mandrágora. Universidade Metodista de São Paulo, 13 (13), pp. 15-21. Doi: 10.15603/2176-0985/ mandragora.v13n13p15-21.

Sullins, D. (2006). Gender and Religion: Deconstructing Universality, Constructing Complexity. American Journal of Sociology, 112 (3), pp. 838-880. Doi: 10.1086/507852.

Tenório, F. G. (2004). Um espectro ronda o terceiro setor, o espectro do mercado: Ensaios de gestão social. Ijuí: Unijuí.

Thompson Jr., E. H. (1991). Beneath the status characteristic: Gender variations in religiousness. Journal for the Scientific Study of Religion, 30 (4), pp. 381-394.

Usarski, F. (2010). Religião como sistema normativo: Considerações sistemáticas e exemplificações. Em: A. Soares \& J. Passos (org.) Teologia e Direito: O mandamento do amor e a meta da justiça, (pp. 21-35). São Paulo: Paulinas.

Valadão Júnior, V. M., Herk, A.C. V.\& Medeiros, C. R. O. (2014). Gênero e economia solidária: Um olhar sobre a participação e atuação das mulheres nas organizações do Terceiro Setor em Uberlândia/MG. Revista Gestão \& Planejamento, 15 (2), pp. 321340.

Walter, T. \& Davie, G. (1998). 'The religiosity of women in the modern West' British. Journal of Sociology, 49 (4), pp. 640-660.

West, C. \& Zimmerman, D. (1987). Doing Gender. Gender and Society, 1, pp. 125151. 